\title{
MOLGENGO: Finding novel molecules with desired electronic properties by capitalizing on their global optimization
}

\author{
Beomchang Kang, ${ }^{\dagger}$ Chaok Seok, ${ }^{*, \dagger}$ and Juyong Lee, ${ }^{* \dagger}$ \\ $\dagger$ Department of Chemistry, Seoul National University, 08826, Seoul, Republic of Korea \\ $\ddagger$ Division of Chemistry and Biochemistry, Department of Chemistry, Kangwon National \\ University, 24341, Chuncheon, Republic of Korea \\ E-mail: chaok@snu.ac.kr; juyong.lee@kangwon.ac.kr
}

\section{Example of converting from SMILES string to inte- ger array using context-free grammar}

Here, we use fluorophore coumarin for example.

SMILES of coumarin is $\mathrm{C} 1=\mathrm{CC}=\mathrm{C} 2 \mathrm{C}(=\mathrm{C} 1) \mathrm{C}=\mathrm{CC}(=\mathrm{O}) \mathrm{O} 2$.

It is converted to 300-digit integer array as as follows:

$[0,1,1,2,1,1,2,1,2,0,1,1,1,1,0,0,1,0,1,1,0,1,1,1,1,1,1,1,0,1,2,1,1,1,1$, $1,0,1,1,1,1,0,0,0,1,1,1,0,1,1,2,1,1,1,1,1,0,0,1,3,1,1,3,1,0,1,80,10,173$, $62,6,80,203,51,157,204,225,74,201,210,156,52,97,65,135,112,132,0,221,98,215$, $149,117,180,169,93,194,240,15,199,187,136,16,156,188,93,102,125,183,12,130$, $222,70,78,7,135,10,159,210,35,0,178,171,240,141,162,199,252,213,72,161,123$, $48,47,12,142,121,223,83,194,25,17,253,145,81,96,233,176,23,189,225,51,235$, $97,220,211,6,5,60,11,185,75,106,175,64,14,196,251,136,27,50,17,81,139,181$, 
$235,133,32,155,16,6,31,62,9,163,155,49,157,170,185,196,252,70,151,37,10,90$, $175,67,74,112,7,85,119,242,155,164,44,214,30,254,238,131,10,85,233,112,30$, 121, 37, 226, 23, 177, 207, 167, 64, 225, 255, 15, 197, 21, 144, 65, 138, 214, 206, 102, 195, 1, 55, 240, 190, 116, 139, 91, 16, 89, 121, 226, 130, 138, 133, 72, 151, 27, 144, 176, 102, 126, 71, 18, 242, 92, 60, 224, 5, 201, 81, 187, 35, 188, 16, 44, 94, 163, 198, 237, 54, 62, 157, 183, 35, $223,24,24,232,243,221,136,35,184,19,236,253,187,80,234,35,226,234]$

\section{RDkit $^{1}$ Molecular Descriptors used for LGBM train- ing}

Morgan fingerprint density 1, 2, 3, Molecular weight, Heavy atom weight, Max absolute partial charge, Max partial charge, Min absolute partial charge, Min partial charge, Number of radical electrons, Number of valence electrons, Fraction of SP3 carbon, kappa shape index 1, 2, 3, Labute ASA, Number of aliphatic carbocycles, Number of aliphatic heterocycles, Number of aliphatic rings, Number of amide bonds, Number of aromatic carbocycles, Number of aromatic heterocycles, Number of aromatic rings, Number of stereocenters, Number of bridgehead atoms, Number of HB acceptors, Number of HB donor, Number of heteroatoms, Number of heterocycles, Number of Lipinski HB acceptors, Number of Lipinski HB donors, Number of rings, Number of rotatable bonds, Number of saturated carbocycles, Number of saturated heterocycles, Number of saturated rings, Number of spiroatoms, Number of unspecified atom stereocenters, Topological SA. 


\section{Excitation energy Distribution of PubChemQC}

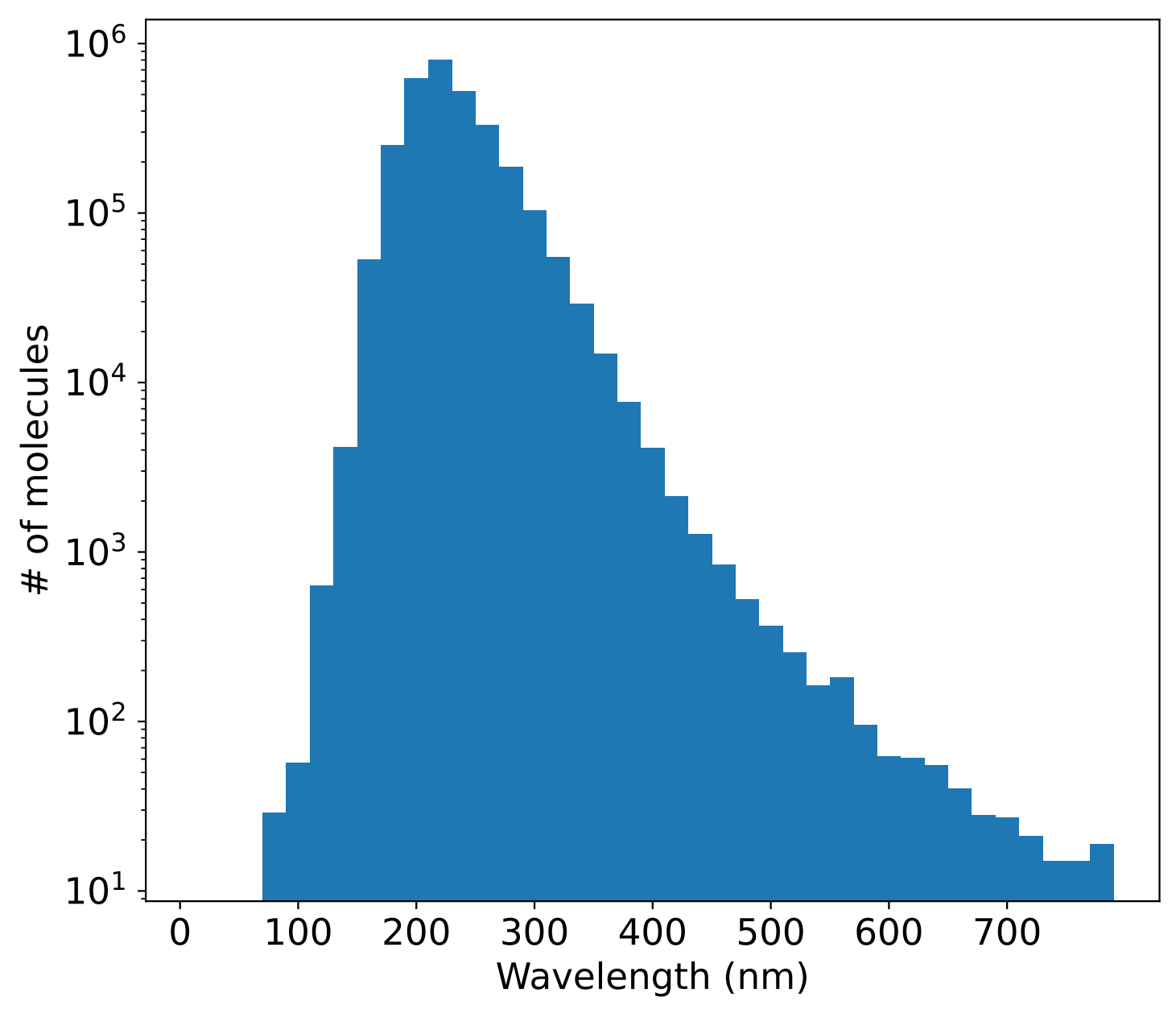

Figure S1: Excitation energy distribution of PubChemQC ${ }^{2}$ 


\section{Validation of HOMO-LUMO optimization results us- ing TD-DFT calculations}
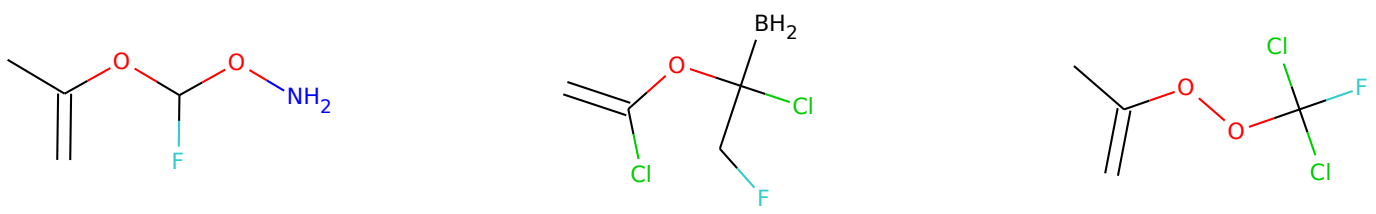

(a) $\quad \lambda_{\text {HOMO-LUMO }}=192.3,(b) \quad \lambda_{\text {HOMO-LUMO }}=\quad 202.3,(\mathrm{c}) \quad \lambda_{\text {HOMO-LUMO }}=\quad 203.6$, $f_{\mathrm{HOMO}-\mathrm{LUMO}}=0.145$ $f_{\mathrm{HOMO}-\mathrm{LUMO}}=0.287$

$f_{\mathrm{HOMO}-\mathrm{LUMO}}=0.841$

Figure S2: Novel molecules found by MOLGENGO with $\lambda_{\text {HOMO-LUMO,target }}=200 \mathrm{~nm}$. TDDFT results of $\lambda_{\text {HOMO-LUMO }}$ and $f_{\text {HOMO-LUMO }}$ are represented below the molecular structures.
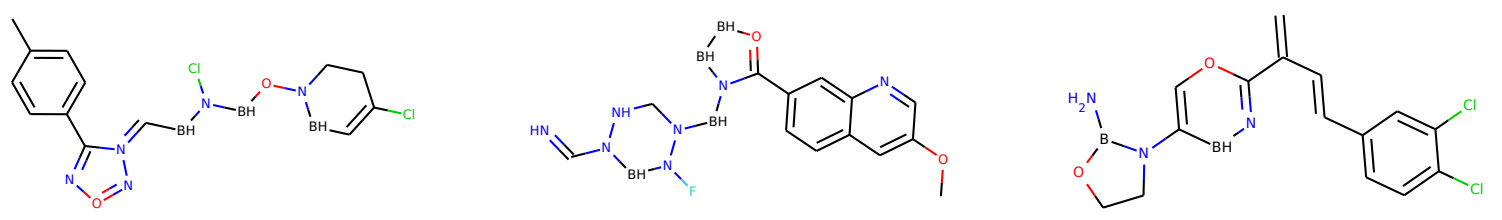

(a) $\quad \lambda_{\text {HOMO-LUMO }}=398.3,(\mathrm{~b}) \quad \lambda_{\text {HOMO-LUMO }}=\quad 400.3,(\mathrm{c}) \quad \lambda_{\mathrm{HOMO}-\mathrm{LUMO}}=396.5$, $f_{\mathrm{HOMO}-\mathrm{LUMO}}=0.974 \quad f_{\mathrm{HOMO}-\mathrm{LUMO}}=1.240 \quad f_{\mathrm{HOMO}-\mathrm{LUMO}}=0.220$

Figure S3: Novel molecules found by MOLGENGO with $\lambda_{\text {HOMO-LUMO,target }}=400 \mathrm{~nm}$. TDDFT results of $\lambda_{\text {HOMO-LUMO }}$ and $f_{\mathrm{HOMO}-\mathrm{LUMO}}$ are represented below the molecular structures. 

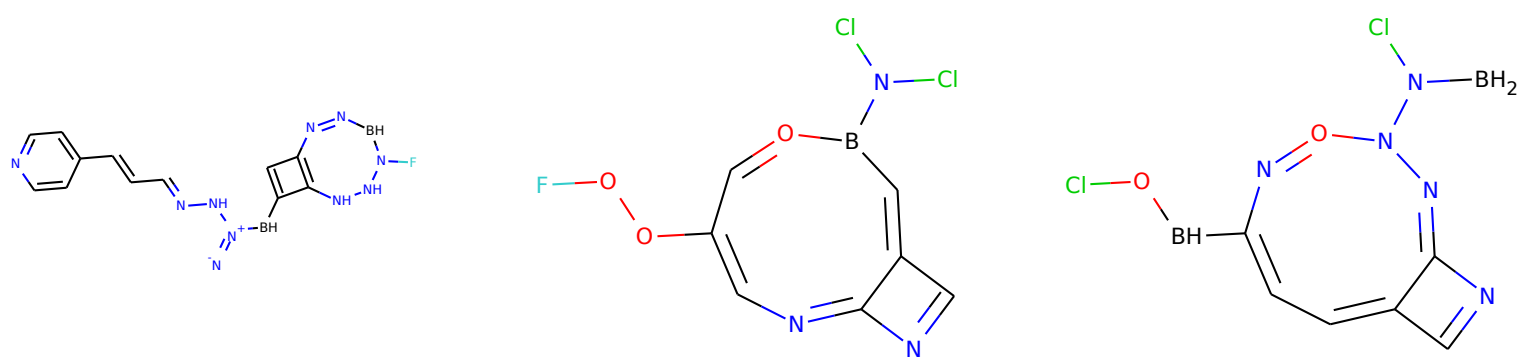

(a) $\quad \lambda_{\text {HOMO-LUMO }}=586.8,(\mathrm{~b}) \quad \lambda_{\text {HOMO-LUMO }}=566.1,(\mathrm{c}) \quad \lambda_{\text {HOMO-LUMO }}=579.6$, $f_{\mathrm{HOMO}-\mathrm{LUMO}}=2.485$

$f_{\mathrm{HOMO}-\mathrm{LUMO}}=0.205$

$f_{\mathrm{HOMO}-\mathrm{LUMO}}=0.171$

Figure S4: Novel molecules found by MOLGENGO with $\lambda_{\text {HOMO-LUMO,target }}=600 \mathrm{~nm}$. TDDFT results of $\lambda_{\text {HOMO-LUMO }}$ and $f_{\text {HOMO-LUMO }}$ are represented below the molecular structures.

\section{References}

(1) Landrum, G. RDKit: Open-Source Cheminformatics Software. 2016,

(2) Nakata, M.; Shimazaki, T. PubChemQC Project: A Large-Scale First-Principles Electronic Structure Database for Data-Driven Chemistry. J. Chem. Inf. Model. 2017, 57, $1300-1308$. 